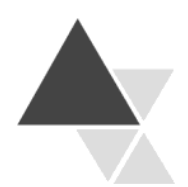

\title{
A Segurança Alimentar no Brasil: Proposição e Usos da Escala Brasileira de Medida da Insegurança Alimentar (EBIA) de 2003 a 2009
}

\begin{abstract}
Ana Maria Segall-Corrêa ${ }^{1}$, Letícia Marin-Leon ${ }^{2}$
Mesmo com os avanços observados na diminuição das desigualdades sociais no Brasil, ainda é grande o contingente de pessoas que vivem em situação de insegurança alimentar. Este trabalho descreve os resultados do uso da Escala Brasileira de Medida da Insegurança Alimentar a partir do seu processo de validação até os inquéritos de abrangência nacional, sua utilização por gestores municipais e, também, para produção acadêmica em diversas universidades. Por fim aponta a relevância da EBIA, como um instrumento auxiliar das políticas públicas de combate à fome no Brasil. Esse trabalho descreve o uso de métodos mistos de investigação, para a validação da EBIA, métodos qualitativos (painéis de especialistas e grupos focais) e quantitativos (inquéritos amostrais de população), em estudo multicêntrico, que envolveu quatro regiões do país, áreas rurais e urbanas e 6 instituições de pesquisa, 5 nacionais e uma norte americana. O principal resultado deste trabalho foi conseguir disponibilizar para os gestores públicos uma ferramenta, a Escala Brasileira de Medida da Insegurança Alimentar e Fome no Brasil - EBIA. Isto possibilitou a realização do primeiro diagnóstico nacional de acesso à alimentação em termos de qualidade e quantidade, bem como, mostrou a existência, em 2004 de 14 milhões de brasileiros que conviveram com a fome nos 3 meses que antecederam as entrevistas da PNAD 2004. Análise complementar dos dados da PNAD 2004, sobre o impacto na população de beneficiários dos programas de transferência de renda apontou que a cada 10 Reais transferidos havia um aumento de $8 \%$ de chance, do domicílio, passar da situação de insegurança alimentar para a de segurança. O processo de investigação que culminou com o desenvolvimento de uma ferramenta (EBIA) para diagnóstico da situação de segurança e insegurança alimentar no domicílio ultrapassou os interesses exclusivamente acadêmicos. Essa experiência constitui exemplo de parceria bem sucedida entre a academia e as instituições públicas do país. A escala tem sido apropriada pelos gestores públicos, de todos os níveis, para identificar populações vulneráveis, avaliar e ajustar os seus programas e ações.
\end{abstract}

Palavras-chave: fome, segurança alimentar, multidisciplinaridade, inquéritos, escala.

\footnotetext{
${ }^{1}$ Professora Associada do Departamento de Medicina Preventiva e Social, Universidade Estadual de Campinas. Endereço para correspondência: Rua Tessália Vieira de Camargo 126, Campinas-SP. Cep13083-887.E-mail: segall@ffcm.unicamp.br

${ }^{2}$ Médico de Saúde Pública do Departamento de Medicina Preventiva e Social, Universidade Estadual de Campinas.

E-mail:letícia@fcm.unicamp.br
} 


\title{
Food Security in Brazil: the proposal and application of the Brazilian Food Insecurity Scale from 2003 to 2009
}

\begin{abstract}
Although Brazil has shown improvement in reducing social inequalities it still has large population living in food insecurity condition. This report describes the validation process developed to obtain the Brazilian Scale of Food Insecurity and Hunger-EBIA, and also shows the results of its use in national household surveys, besides it describes its use by several universities and municipalities, in an attempt to demonstrate EBIA's relevance as an ancillary tool for public policies towards fighting hunger in Brazil. Qualitative and quantitative techniques were combined to validate the EBIA. Panels of expert authorities and focus groups were used besides population sampling surveys, conducted in multicentric settings. This study was conducted in four regions in the Country and included rural and urban areas reached by six research institutions of which five were Brazilian and one was in the US. The main result was the development of the EBIA and its use by police makers. This allowed the first national diagnosis of the state of food access with regards to quantity and quality, as well as pointed out the existence of 14 million Brazilians living with hunger, in the three months that preceded the National Household Sampling Survey of 2004 PNAD. A complimentary analysis of the impact of income transfer programs showed that for each transfer of $\mathrm{R} \$ 10.00$ there was an increase of $8 \%$ chance for the food insecure households to trespass to food security. The investigation ended up in the development of EBIA as a tool for evaluating the household food security that went beyond the exclusive academic interests. This constituted an example of well succeeded partnership between the academic research and the country public policies.
\end{abstract}

Key words: hunger, food security, multidisciplinarity, survey, scale.

\section{Introdução}

A partir do final da segunda guerra mundial, a questão de segurança alimentar (S.A.) das populações era compreendida, basicamente, como uma limitação de disponibilidade de alimentos e uma ameaça aos países, especialmente os europeus que se encontravam, imediatamente após esse conflito, sem condições de produção de alimentos em quantidade suficiente para sua população. Essa primeira compreensão da S.A. levou o Fundo das Nações Unidas para a Alimentação (FAO), a propor um indicador de medida padronizado a partir da disponibilidade calórica per capita, com a finalidade de acompanhar tendências históricas e estabelecer comparações entre os países ${ }^{[1,2]}$.

Progressivamente e à medida que o conceito de segurança alimentar ganhava complexidade, outros indicadores foram sendo introduzidos visando captar e mensurar as novas dimensões da segurança alimentar incorporadas ${ }^{[3]}$. Para avaliar as condições de acesso aos alimentos passou-se a utilizar o indicador de rendimento familiar, o gasto familiar com a compra dos alimentos e o consumo alimentar individual, por meio de sua freqüência semanal ou de análise da ingestão de alimentos das últimas 24 horas. Os indicadores antropométricos tradicionalmente estimam a magnitude da insegurança alimentar pela sua conseqüência nutricional, especialmente entre crianças ${ }^{[1]}$. Todos eles, incluindo a disponibilidade calórica per capita, apresentam vantagens e desvantagens, considerando os objetivos das estimativas para as quais eram adotados. $\mathrm{Na}$ sua maioria são recursos indiretos de análise da magnitude da segurança ou insegurança alimentar ${ }^{[4]}$. Com exceção do consumo alimentar, os demais indicadores são especificamente apropriados para identificar e analisar os determinantes da insegurança alimentar ou dimensionar suas conseqüências para a saúde e nutrição dos indivíduos e populações. 
Somente no início dos anos 90 do século XX, passos importantes foram dados no sentido de investigar indicadores diretos e com validade aceitável para medir a segurança alimentar familiar. Dois projetos de investigação, nos Estados Unidos da América, deram base à elaboração de uma escala psicométrica para medir de forma direta a magnitude da segurança ou insegurança alimentar da população. O primeiro era uma tese de doutorado da Universidade de Cornell [5,6] e o outro, uma escala que objetivava identificar famílias em situação de fome para inclusão em projeto de intervenção e alertar a sociedade norte americana sobre o problema da insegurança alimentar ${ }^{[7]}$. A junção destas duas experiências permitiu o desenvolvimento de um instrumento de medida com 18 itens, o Household Food Security Survey Module HFSSM, ${ }^{[8]}$ compondo uma escala capaz, não apenas de mensurar a magnitude do problema da insegurança alimentar na população, mas, também, de identificar diferentes graus de acesso aos alimentos: desde a completa satisfação das necessidades alimentares (Segurança Alimentar), até as experiências em níveis de progressiva gravidade de restrição de alimentos (Insegurança Alimentar Leve, Moderada e Grave) ${ }^{[8,9]}$. Segundo Radimer e Olson, [6] essa progressão reflete a compreensão de que, inicialmente, há a preocupação ou incerteza quanto a capacidade pessoal ou familiar de obter os alimentos necessários para o futuro próximo; em seguida, vem o comprometimento da qualidade da alimentação, como uma estratégia de garantia da quantidade necessária; e, por último, se os problemas que originaram as dificuldades de acesso não se resolvem, ocorre a redução da quantidade, inicialmente para os adultos da família e, na situação mais grave, para as crianças.

Essa escala tem sido usada para acompanhar a evolução dos níveis de segurança alimentar nos Estados Unidos, desde o início dos anos $90^{[9,10]} \mathrm{e}$, em países menos desenvolvidos, a partir de sua adaptação e validação, para as realidades locais $[11,12,13,14]$. Sua utilização permitiu avançar das estimativas indiretas da insegurança alimentar, para o dimensionamento direto das deficiências quantitativas e qualitativas de acesso aos alimentos.

Ao analisar as desvantagens dos indicadores tradicionais e as vantagens para a mensuração do fenômeno da segurança alimentar conferidas pela HFSSM pesquisadores reunidos na UNICAMP, em 2003, discutiram a pertinência política e viabilidade técnica de adaptar e validar, esse instrumento para a realidade brasileira. Identificou-se nesta ocasião que a academia poderia fornecer contribuição relevante no sentido de incorporar às políticas do Fome Zero (FZ), que acabava de ser lançado ${ }^{[1]}$, indicadores diretos de quantificação da população sujeita a diferentes graus de insegurança alimentar, apropriados, também, para o acompanhamento e avaliação de suas ações e estratégias. Na busca de identificação da população vulnerável à IA ou fome e, conseqüentemente, de seleção dos indivíduos elegíveis para participação nos programas sociais de transferência de renda, os formuladores do FZ basearam-se em indicadores de rendimento familiar per capita. Estimaram, àquela ocasião, a existência de 46 milhões de pessoas vulneráveis à insegurança 
alimentar ${ }^{[1]}$. O uso de indicadores de renda, referidos ou não às linhas de pobreza e indigência, forneciam diferentes estimativas de população alvo dos programas do $\mathrm{FZ}$, a depender do método usado, o que constituía uma dificuldade operacional importante. Por todas essas razões era pertinente a proposição de uma escala de medida direta da insegurança alimentar no domicílio.

O objetivo desta comunicação é o de descrever a relevância da investigação acadêmica para as políticas públicas do país, usando para isso o exemplo dos desdobramentos ocorridos entre a disponibilização de indicadores de segurança alimentar e os resultados em âmbitos locais e nacional.

\section{Métodos para o desenvolvimento da Escala Brasileira de Medida da Insegurança Alimentar - EBIA}

No Brasil, a investigação para o desenvolvimento da EBIA empregou inicialmente métodos qualitativos, seguidos de pré-testes e testes em inquéritos populacionais quantitativos ${ }^{[15,16]}$. Contou, nesse processo, com participação de seis instituições de pesquisa: Universidade Estadual de CampinasUNICAMP (coordenação), Universidade de Brasília-UNB, Universidade Federal da Paraíba-UFPB, Universidade Federal do Mato Grosso-UFMT e Instituto Nacional de Pesquisa da Amazônia-INPA, e da Universidade de Connecticut-UCCON-USA.
A fase qualitativa do estudo constituise, inicialmente, no que pode ser chamado de validade de face: a avaliação, por grupo de especialistas em nutrição e em gestão pública de programas sociais, da proposta de adequação à realidade local da escala traduzida para o português; e também, da pertinência técnica e política do uso de um modelo de escala originário de população social e culturalmente diferente da brasileira. $\mathrm{O}$ grupo de especialistas e de gestores considerou a escala proposta adequada para o diagnóstico de segurança alimentar, acompanhamento e avaliação das políticas públicas. Foram geradas, na reunião desse grupo, algumas sugestões sobre o processo de validação de conteúdo em grupos focais, adequação de linguagem e redução do tempo de referência dos eventos de interesse de 12 meses para 3 meses anteriores à entrevista. Foi sugerido, também, transformar a estrutura da escala de alguns itens afirmativos para o formato de um questionário. Os especialistas sugeriram os indicadores de renda, escolaridade e consumo alimentar da pessoa entrevistada como necessários para a validação externa da escala. Propuseram reduzir os itens da escala original de 18 itens para 15 e mudaram, também, a forma de referência da freqüência de cada item da escala.

Os grupos focais (GF), em número de 11, organizados em cidades e áreas rurais de quatro macro regiões do país (Norte, Nordeste, Centro-Oeste e Sudeste) contavam com 10 a 12 participantes, selecionadas em comunidades pobres, o que possibilitaria incluir nas discussões temas relacionados à 
experiência dos moradores com a insegurança alimentar e fome. Os grupos focais começavam sempre com a abordagem sobre segurança alimentar, a experiência de vida de cada um e, em seguida, eram colocados na roda os conteúdos centrais da escala, para depois disso serem analisadas, as estruturas de cada item e a adequação da linguagem para cada local ${ }^{[19,20]}$.

Em síntese, chamou atenção dos pesquisadores o grau de entendimento e a complexidade das reflexões sobre os temas discutidos que eram verbalizados pela maioria dos participantes dos grupos focais. Alguns exemplos estão nas falas a seguir ${ }^{[17]}$ :

"Não tenho segurança que no fim do mês eu vou ter dinheiro pra fazer compra."

"Não temos alimentos suficientes o tempo todo. Quando não temos inteiramos com a farinha."

"Comer apenas um tipo de alimento, mesmo ele sendo saudável, não é alimentação saudável. Nós passamos 15 dias comendo só banana."

"Primeiro o aluguel, depois o transporte, a luz e água, só depois vem o alimento."
"As familias fazem assim..., diminuem a alimentação, os pais comem menos pra deixar para os filhos e vão tentando manter o estoquinho pequeno."

"A fome dói, panela virada, geladeira sem nada, nem ovo para suprir o estômago."

"Acho que a coisa mais triste é a fome...; é a pior das violências; eu já passei, eu sei como é triste."

"Eu acho que qualquer cidadão tinha que ter o suficiente pra se manter; Ter emprego, poder sustentar sua família."

Os integrantes dos grupos focais entenderam ser útil a existência de um instrumento de avaliação de insegurança alimentar e fome, para que a "situação da população ficasse clara para os políticos e autoridades". Fizeram sugestões de adequação de linguagem, julgaram a pertinência dos 3 meses para lembrança dos eventos e avaliaram que uma única escala poderia ser aplicada, tanto em área urbana quanto rural (Quadro 1). Detalhes destes procedimentos metodológicos podem ser encontrados em outras publicações do grupo de pesquisa ao qual pertencem as

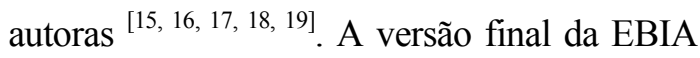
encontra-se anexada a este. 


\section{Quadro 1. Itens das escalas de medida domiciliar da segurança alimentar, versão norte americana original e versão adaptada para o Brasil}

\section{$\operatorname{HFSSM}(U S A) *$}

We worried whether our food would run out before we got money to buy. more. Was that often, sometimes, or never true for you in the last 12 months?

The food that we bought just didn't last and we didn't have money to get more. Was that often, sometimes, or never true for you in the last 12 months?

We couldn't afford to eat balanced meals." Was that often, sometimes, or never true for you in the last 12 months?

In the last 12 months, did you or other adults in the household ever cut the size of your meals or skip meals because there wasn't enough money for food? (Yes/No)

In the last 12 months, did you ever eat less than you felt you should because there wasn't enough money for food? (Yes/No)

In the last 12 months, were you ever hungry, but didn't eat, because you couldn't afford enough food? (Yes/No)

In the last 12 months, did you lose weight because you didn't have

enough money for food? (Yes/No)

In the last 12 months did you or other adults in your household ever not eat for whole day because there wasn't enough money for food? (Yes/No)

We relied on only a few kinds of low-cost food to feed our children because we were running out of money to buy food." Was that often, sometimes, or never true for you in the last 12 months?

We couldn't feed our children a balanced meal, because we couldn't afford that." Was that often, sometimes, or never true for you in the last 12 months?

\section{EBIA - Versão preliminar**}

Nos últimos 3 meses, a Sra. teve a preocupação de que a comida na sua casa acabasse antes que Sra.tivesse condição de comprar mais comida?

Nos últimos 3 meses, a comida acabou antes que a Sra. tivesse dinheiro para comprar mais?

Nos últimos 3 meses, a Sra. ficou sem dinheiro para ter uma alimentação saudável e variada?

Nos últimos 3 meses, a Sra.ou algum adulto em sua casa diminuiu, alguma vez, a quantidade de alimentos nas refeições ou pulou refeições, porque não havia dinheiro suficiente para comprar a comida?

Nos últimos 3 meses, a Sra. alguma vez comeu menos do que achou que devia porque não havia dinheiro o suficiente para comprar comida?

Nos últimos 3 meses, a Sra. alguma vez sentiu fome mas não comeu porque não podia comprar comida suficiente?

Nos últimos 3 meses, a Sra. perdeu peso porque não tinha dinheiro suficiente para comprar comida?

Nos últimos 3 meses, a Sra. ou qualquer outro adulto em sua casa ficou, alguma vez, um dia inteiro sem comer ou, teve apenas uma refeição ao dia, porque não havia dinheiro para comprar a comida?

Nos últimos 3 meses, a Sra. teve que se arranjar com apenas alguns alimentos para alimentar os moradores com menos de 18 anos, porque o dinheiro acabou?

Nos últimos 3 meses, a Sra. não pode oferecer a algum morador com menos de 18 anos, uma alimentação saudável e variada, porque não tinha dinheiro? 
The children were not eating enough because we just couldn't afford enough food." Was that often, sometimes, or never true for you in the last 12 months?

In the last 12 months, did you ever cut the size of any of the children's meals because there wasn't enough money for food?

In the last 12 months, were the children ever hungry but you just couldn't afford more food?

In the last 12 months, did any of the children ever skip a meal because there wasn't enough money for food?

In the last 12 months did any of the children ever not eat for a whole day because there wasn't enough money for food?
Nos últimos 3 meses, algum morador com menos de 18 anos não comeu em quantidade suficiente, porque não havia dinheiro para comprar a comida?

Nos últimos 3 meses, a Sra., alguma vez, diminuiu a quantidade de alimentos das refeições de algum morador com menos de 18 anos, porque não havia dinheiro suficiente para comprar a comida?

Nos últimos 3 meses, algum morador com menos de 18 anos teve fome, mas a Sra. simplesmente não podia comprar mais comida?

Nos últimos 3 meses, alguma vez algum morador com menos de 18 anos deixou de fazer alguma refeição, porque não havia dinheiro para comprar comida?

Nos últimos 3 meses, algum morador com menos de 18 anos ficou sem comer por um dia inteiro, porque não havia dinheiro para comprar comida?

* Algumas perguntas são afirmativas e seguidas da frequência de ocorrência outras são perguntas com respostas Sim ou Não. Outras 3 perguntas complementares e referentes a outras dificuldades de acesso aos alimentos foram excluídas da versão brasileira.

** As perguntas brasileiras eram todas seguidas das freqüências: em quase todos os dias; em alguns dias; em apenas 1 ou 2 dias; não sabe ou recusa responder, exceto para perda de peso, que as alternativas são: pouca; média; muita; não sabe ou recusa responder. A EBIA sofreu pequenas alterações necessárias para adequação ao questionário da PNAD, após novo teste em campo.

A segunda etapa, quantitativa, do processo de validação da EBIA se deu com a realização de inquéritos em amostras intencionais da população urbana e das áreas rurais das 4 macro regiões do Brasil. Buscouse com estas escolhas aplicar a escala proposta em contextos de diversidade social e de hábitos culturais, especialmente alimentares, que são observados no Brasil e obter, com estes procedimentos, a validade externa, preditiva, dos itens da escala ${ }^{[15,16,18]}$. Foram

selecionadas amostras em áreas próximas das localidades onde foram realizados os grupos focais, com cerca de 200 domicílios em cada área rural e urbana, totalizando, ao final do processo de teste da escala, mais de 1800 domicílios. Essa amostra era composta por estratos sociais de classe média, média baixa, pobres e muito pobres, o que permitiu analisar a correlação dos gradientes de segurança alimentar com diferenças de renda, além dos testes com outras variáveis socioeconômicas [16].

A validação da EBIA possibilitou contemplar as especificidades e diversidades nacionais, resultando, em instrumento de medida aplicável à população brasileira, seja ela rural ou urbana. A EBIA é um instrumento de medida de alta validade interna e externa como ficou demonstrado com o uso de 
diferentes e sofisticados procedimentos analíticos $^{[15,16,20,21,22]}$.

É importante registrar que todas as atividades, tanto nos seus aspectos qualitativos quanto quantitativos, foram acompanhadas por avaliadores externos reunidos em duas oficinas de trabalho organizadas, independentemente dos pesquisadores envolvidos, por profissionais da organização Pan-Americana da Saúde e realizadas nas dependências de sua sede em Brasília.

A estrutura da EBIA com suas 15 perguntas, cuja síntese está no quadro 1, constitui agrupamentos conceituais que

Quadro 2. Pontuação para classificação dos domicílios nas categorias de segurança alimentar

\begin{tabular}{|lll|}
\hline & \multicolumn{2}{l|}{ NÚMERO DE PONTOS } \\
\cline { 2 - 3 } & Famílias com menores de & Famílias sem menores de \\
& 18 anos & 18 anos \\
\hline Segurança Alimentar & 0 & 0 \\
Insegurança Alimentar Leve & 1 a 5 & 1 a 3 \\
Insegurança Alimentar Moderada & 6 a 10 & 4 a 6 \\
Insegurança Alimentar Grave & 11 a 15 & 7 a 8 \\
\hline
\end{tabular}

\section{Aplicação e usos da escala de medida da Segurança Alimentar no Brasil}

A EBIA tem sido amplamente usada no Brasil, ora com propósitos acadêmicos, atestando sua relevância para a formação de recursos humanos comprometidos com os esforços de combater as desigualdades sociais no nosso país, ora como indicador adequado e válido para as políticas de combate à insegurança alimentar e fome, tanto em âmbito federal quanto municipal. permitem estimar as prevalências de segurança alimentar e classificar os domicílios em quatro níveis: com Segurança Alimentar, em Insegurança Alimentar Leve, Moderada ou Grave. Para essa classificação foi estabelecido um algoritmo que atende aos pontos de cortes mostrados no quadro 2. As pontuações para domicílios com crianças são diferentes daquelas usadas para classificar os domicílios onde residem apenas adultos, porque nesses são utilizadas apenas oito das quinze perguntas da escala. 
segundo uma dissertação de mestrado (UNB). Estes inquéritos populacionais confirmaram a validade, tanto interna quanto externa da EBIA e mostraram os primeiros resultados em grandes populações. Foram encontrados valores de prevalências muito próximos e condizentes com as semelhanças entre as populações das duas cidades. Era de cerca de $60 \%$ a proporção de domicílios com garantia de acesso aos alimentos em quantidade e qualidade adequados (segurança alimentar). Sendo de $6,6 \%$ e $7,6 \%$ a proporção em Campinas e Brasília, respectivamente, de insegurança alimentar grave, ou seja, com restrição quantitativa importante de alimentos, permitindo concluir pela existência de fome nesses domicílios, inclusive entre as suas crianças.

A partir daí, a EBIA passou a ser considerada instrumento importante para a geração de indicador direto de medida domiciliar de segurança alimentar. Além de relevante, no Brasil, para o monitoramento da IA, para avaliação dos efeitos das políticas públicas setoriais de combate à insegurança alimentar e análise dos eventos sociais ou econômicos geradores de impacto no acesso da população aos alimentos.

Em 2004 a EBIA foi incorporada, por decisão do Ministério do Desenvolvimento Social e Combate à Fome, à Pesquisa Nacional por Amostra de Domicílios-PNAD2004. Surgiu assim, o primeiro diagnóstico, no Brasil, de segurança e insegurança alimentar domiciliar, com abrangência e representatividade nacionais ${ }^{[25]}$. Os resultados mostraram que cerca de $40 \%$ da população brasileira convivia com algum grau de insegurança alimentar, sendo $18 \%$ com IA
Leve, outros $14,1 \%$ com IA moderada e 7,7\% com IA Grave. $O$ que significava aproximadamente a 14 milhões de brasileiros convivendo com a fome e outros 25 milhões com restrição quantitativa importante na sua alimentação, totalizando mais 39 milhões de brasileiros. A PNAD2004 deixou clara, considerando os resultados oriundos do uso da EBIA, a mesma desigualdade entre as regiões, já apontada por indicadores sociais, econômicos e de saúde. Análises complementares apontaram que, em cada uma das regiões do país, as situações mais críticas eram encontradas nos domicílios com os mais baixos rendimentos, com precárias condições de saneamento, tendo as mulheres como responsáveis pela família, onde residiam crianças pequenas ou adolescentes e, onde o responsável pela família possuía baixa escolaridade ${ }^{[19,26]} \mathrm{O}$ rendimento familiar per capita abaixo de um quarto do salário mínimo da época, por exemplo, aumentava a prevalência de IA grave em 15 vezes, comparativamente a rendimentos superiores a meio salário mínimo per capita. ${ }^{[19]}$

As informações da PNAD2004 mostraram ainda, que os usuários do programa Bolsa Família (BF) apresentavam prevalências mais altas $(14,9 \%)$ de insegurança alimentar grave, mais que o dobro da observada na média nacional ${ }^{[25]}$. Isto levantou, à ocasião, polêmica com algumas hipóteses nos jornais diários sobre uma suposta baixa eficiência do programa. Entendeu-se, entretanto, como mais plausível, que a população de usuários concentrava condições outras de vulnerabilidade que reduziam o impacto da transferência de renda. Essa hipótese foi confirmada ao ser analisada, por meio de modelagem estatística adequada, as 
mudanças ocorridas no perfil de segurança alimentar a partir do acréscimo de renda oriundo do programa ${ }^{[27]}$. Observou-se que a cada 10 reais de acréscimo na renda familiar, por meio do BF, a chance de segurança alimentar aumentava em $8 \%$. Isto significa que a cada 60 reais, perto do valor médio de transferência à época, uma chance de cerca de $50 \%$ de mudança de status, passando o domicílio de situação de insegurança para a segurança alimentar.

Em 2006, outro inquérito de abrangência nacional, a Pesquisa Nacional de Demografia e Saúde da Mulher e da Criança PNDS2006 ${ }^{[28]}$ também foi acrescida de um módulo sobre segurança alimentar, contendo a EBIA, por exigência de edital da Secretaria de Ciência e Tecnologia e Insumos Estratégicos do Ministério da Saúde ${ }^{[2]}$. As análises resultantes e relativas aos domicílios onde residia pelo menos uma mulher em idade fértil mostraram que a magnitude da insegurança alimentar ainda era grande no Brasil, a despeito das políticas públicas específicas, como o Bolsa Família e da redução da proporção de população pobre e extremamente pobre ${ }^{[29,30,31]}$. Era de 9,7\% a prevalência de domicílios com moradores experimentando insegurança alimentar moderada (restrição quantitativa de alimentos entre os adultos) e de 4,8\% a insegurança grave, caso em que a restrição alimentar atingia, também, as crianças desses domicílios. As mesmas condições de vulnerabilidade observadas na PNAD2004 foram também detectadas na PNDS2006. A insegurança alimentar em qualquer dos seus graus de gravidade eram mais freqüentes nos domicílios dos estratos sociais mais baixos, nos domicílios com crianças e onde o responsável era uma mulher ou de baixa escolaridade.

No país, vários outros inquéritos populacionais ou estudos específicos foram realizados, em várias regiões, nos anos seguintes ao da validação da EBIA, ${ }^{[32,33]}$ tanto por solicitação de gestores municipais dos programas sociais quanto por interesses dos pesquisadores, que reconheceram na EBIA uma possibilidade de melhor compreensão do fenômeno da fome e nova oportunidade de parceria das universidades com a gestão da política pública (Quadros 3a e 3b).

Em 2005 foi constituída a Rede Interinstitucional e Multidisciplinar de Investigação em Segurança Alimentar- Rede Alimenta, inicialmente com os pesquisadores das várias instituições que participaram do desenvolvimento da EBIA e hoje ampliada por novos integrantes. A Rede Alimenta é fruto dos trabalhos de validação da EBIA, mas é, também, a grande incentivadora de várias das investigações incluídas nos Quadros 3a e 3b. Seus pesquisadores têm buscado aprimorá-la e, também, adaptá-la para uso em grupos específicos, como os indígenas ${ }^{[34,35]}$ e outras populações que vivem à margem da sociedade nacional. Alguns de seus projetos envolvem ainda o estudo de indicadores complementares que poderão trazer maior compreensão do fenômeno de segurança alimentar. 
Quadro 3a. Uso da EBIA em investigações de interesse e demanda das políticas publicas em âmbito municipal e federal entre 2003-2009

\begin{tabular}{|llll|}
\hline Município & Ano & Amostra & Publicação \\
\hline Americana & 2005 & 431 domicílios & Relatório Técnico \\
\hline Prefeitura de Suzano & 2007 & 431 domićílios & Relatório Técnico \\
\hline $\begin{array}{l}\text { UNIChapecó- } \\
\text { 32 municípios }\end{array}$ & $2004 / 2005$ & 10.000 domicílios & Relatório Técnico \\
\hline Paraiba & $2004 / 2006$ & 14 municípios & Revista de Nutrição \\
\hline PNAD 2004 & 2004 & 112.329 domicílios & Brasil - IBGE \\
\hline PNDS 2006 & 2006 & 13.056 domicílios & Brasil-CEBRAP-MS \\
\hline PNAD 2009 & Em curso & 139.000 domicílios & Brasil - IBGE \\
\hline IBASE & 2007 & 5.000 domicílios & $\begin{array}{l}\text { Revista Democracia } \\
\text { Viva - IBASE }\end{array}$ \\
\hline
\end{tabular}

A resolução da segunda Conferência Nacional de Segurança Alimentar e Nutricional promovida pelo Conselho Nacional de Segurança Alimentar e Nutricional em 2004, ${ }^{[36]}$ definiu a segurança alimentar como: "o direito de todos ao acesso regular e permanente a alimentos de qualidade, em quantidade suficiente, sem comprometer o acesso a outras necessidades essenciais, tendo como base práticas alimentares promotoras de saúde, que respeitem a diversidade cultural e que sejam social, econômica e ambientalmente sustentáveis".

Esse conceito amplia as concepções mais reconhecidas e agrega as dimensões de acesso aos alimentos como direito humano, bem como a exigência de sua sustentabilidade, social, econômica e ambiental. Essas novas dimensões tornam mais complexos os intentos de mensurar a segurança alimentar, os seus determinantes e os efeitos sobre o bem estar das pessoas e comunidades ${ }^{[3]}$. A inclusão de uma questão relacionada à sustentabilidade na Escala Latino Americana e do Caribe de Medida da Segurança Alimentar (ELCSA) apresentou adequação psicométrica em pesquisa realizada no Haiti, mas não em outros países como o México, por exemplo. Todas estas questões atestam a necessidade de novas investigações sobre indicadores de medida de segurança alimentar, de preferência com abordagem multi e interdisciplinar, o que constitui novos desafios para pesquisadores e gestores das políticas públicas. 
Quadro 3b. Uso da EBIA em investigações acadêmicas

\begin{tabular}{|c|c|c|c|c|}
\hline Instituição & Tipo & $\begin{array}{l}\text { Ano da } \\
\text { pesquisa }\end{array}$ & Amostra & Município \\
\hline UNICAMP-FCM & Doutorado & 2003 & 817 & Campinas (SP) \\
\hline UNICAMP-FCM & Mestrado & 2007 & $\begin{array}{l}\text { Estudo } \\
\text { qualitativo }\end{array}$ & Campinas (SP) \\
\hline $\begin{array}{l}\text { UNICAMP- } \\
\text { FEAGRI }\end{array}$ & Mestrado & 2004 & $\begin{array}{l}300 \text { assentados } \\
\text { de reforma } \\
\text { agrária }\end{array}$ & $\begin{array}{l}\text { Pontal do Paranapanema } \\
\text { (SP) }\end{array}$ \\
\hline $\begin{array}{l}\text { UNICAMP- } \\
\text { FCM/ } \\
\text { FEAGRI }\end{array}$ & $\begin{array}{l}\text { Pesquisa } \\
\text { multicêntrica }\end{array}$ & $2005 / 2007$ & $\begin{array}{l}\text { Área rural- } \\
\text { Insegurança } \\
\text { alimentar e } \\
\text { saúde }\end{array}$ & $\begin{array}{l}\text { Municípios dos estados } \\
\text { de São Paulo, Paraná, } \\
\text { Santa Catarina e Minas } \\
\text { Gerais. }\end{array}$ \\
\hline UNICAMP & Pesquisa & 2007 & 1200 gestantes & Campinas (SP) \\
\hline UNICAMP & Pesquisa & 2006 & 950 Mães & Sumaré (SP) \\
\hline UNICAMP & I. Científica & & & \\
\hline UNESP & Mestrado & 2008 & & \\
\hline USP-FSP & Mestrado & 2007 & 820 crianças & São Paulo \\
\hline INPA-Manaus & Pesquisa & 2006 & $\begin{array}{l}\text { Estudo } \\
\text { qualitativo - } \\
\text { teste da EBIA }\end{array}$ & $\begin{array}{l}\text { Comunidades indígenas } \\
\text { Amazonas }\end{array}$ \\
\hline INPA-Manaus & Pesquisa & 2004 & 194 domicílios & Manaus (AM) \\
\hline UFRJ & Pesquisa & 2004 & $\begin{array}{l}1.085 \\
\text { domicílios }\end{array}$ & Duque de Caxias (RJ) \\
\hline UFRJ & Mestrado & 2007 & 1.085 & Duque de Caxias (RJ) \\
\hline UFRJ & Mestrado & 2007 & 1.085 & Duque de Caxias (RJ) \\
\hline UFRJ & Doutorado & 2008 & 1085 & Duque de Caxias (RJ) \\
\hline UFPE & Doutorado & & 458 & São João do Tigre (PE) \\
\hline UFPB & Mestrado & & & \\
\hline UFMTS & Mestrado & 2007 & 49 famílias & Pop. Teréna (MS) \\
\hline UFRGS & Doutorado & 2004 & Pnad2004 & $\begin{array}{l}\text { Domicílios do estado do } \\
\text { Rio Grande do Sul }\end{array}$ \\
\hline UFMT & Mestrado & 2007 & 700 & Sinop \\
\hline UFMT & Mestrado & 2009 & $\begin{array}{l}\text { Adolescentes - } \\
350\end{array}$ & $\begin{array}{l}\text { Quatro Municípios- } \\
\text { BR163 }\end{array}$ \\
\hline UFMT & Mestrado & 2009 & Crianças - 300 & $\begin{array}{l}\text { Quatro Municípios- } \\
\text { BR163 }\end{array}$ \\
\hline UNB & Mestrado & 2004 & 1860 & Brasília (DF) \\
\hline UNB & Doutorado & 2004 & 112.329-PNAD & Nacional \\
\hline $\begin{array}{l}\text { UFMG- } \\
\text { CEDEPLAR }\end{array}$ & Doutorado & 2004 & $\begin{array}{l}\text { Região NE } \\
\text { (exclusivamente) }\end{array}$ & Nacional \\
\hline ENCE-IBGE & Mestrado & 2004 & 112.329-PNAD & Nacional \\
\hline UEL & Mestrado & 2008 & 421 & Toledo (PR) \\
\hline UFCSPA & Mestrado & 2008 & 313 & Porto Alegre (RS) \\
\hline
\end{tabular}




\section{Conclusão}

$\mathrm{O}$ processo de investigação que culminou com o desenvolvimento da EBIA para mensuração direta da situação de segurança e insegurança alimentar no domicílio ultrapassou os interesses exclusivamente acadêmicos. Essa experiência constitui exemplo de parceria bem sucedida entre a academia e as políticas públicas do país.

A EBIA é reconhecida como um instrumento importante para orientação das políticas públicas de combate à insegurança alimentar e a fome. Isso explica a sua inclusão, como indicador em dois inquéritos nacionais já realizados. Fará parte, também, da PNAD de 2009, que está em campo neste momento, o que possibilitará a análise da evolução da segurança e insegurança alimentar no Brasil de 2004 a 2009. O desenvolvimento da EBIA e seus desdobramentos têm contribuído para a formação de recursos humanos comprometidos com a solução dos problemas sociais e, em especial, aqueles relativos à alimentação e nutrição, que o Brasil ainda enfrenta.

Todas as iniciativas relatadas mostraram resultados consistentes e mais do que isso, alertaram os gestores das políticas públicas para um problema que aparentemente ainda se mantém na sombra, para muitos, escondido no eufemismo de população vulnerável ou de risco de insegurança alimentar. Com a EBIA o baixo acesso das famílias aos alimentos tanto em qualidade quanto em quantidade, voltaram novamente à cena, com visibilidade própria e como condição diretamente mensurável.

\section{Referências Bibliográficas}

[1] Belik W. Perspectivas para segurança alimentar e nutricional no Brasil. Saúde e Sociedade 2003;12:12-20.

[2] Migotto M, Davis B, Carletto G, Beegle K. Measuring Food Security Using Respondents' Perception of Food Consumption Adequacy. ESA Working Paper No. 05-10 2005 [cited Setembro de 2009]; Available from: http://www.fao.org/docrep/008/af286e/af286 $\underline{\mathrm{e} 00 . \mathrm{htm}}$

[3] Kepple A, Segall-Corrêa A. Conceituando e Medindo Segurança Alimentar e Nutricional. Ciência \& Saúde Coletiva (Online) 2008, Agosto [cited 2008 Agosto, 2008]; 11 - 19]. Available from: http://www.abrasco.org.br/cienciaesaudecole tiva/artigos/artigo int.php?id artigo $=1511$

[4] Pérez-Escamilla R, Segall-Corrêa AM. Food Insecurity Measurement and Indicators: A critical review. Rev. de Nutr 2008;21(Suplemento de Segurança Alimentar):15-26.

[5] Radimer K, Campbell K. Development of indicators to assess hunger. Journal of Nutrition 1990;Suppl:1544S-1548S.

[6] Radimer K, Olson C, Greene J, Campbell C, Habicht J-P. Understanding hunger and developing indicators to assess it in women and children. J Nutr Educ 1992;24 Suppl:36-45.

[7] Wehler C, Scott R, Anderson J. The Community Childhood Hunger Identification Project: A model of domestic hunger - demonstration project in Seattle. J Nutr Educ 1992;24 Suppl:29-35.

[8] Bickel G. Guide to Measuring Household Food Security in the United States. Food and Nutrition Service 2000 [cited agosto 2008]; Available from: www.ers.usda.gov/publications/fanrr11$\underline{1 / \text { fanrr11_1b.pdf }}$ 
[9] Nord M, Andrews M, Winicki J. Frequency and duration of food insecurity and hunger in US households. J Nutr Educ Behav. 2002;34(4):194-200.

[10] Frongillo EA. Validation of Measures of Food Insecurity and Hunger. J. Nutr. 1999;129(2):506-.

[11] Coates J, Wilde PE, Webb P, Rogers BL, Houser RF. Comparison of a Qualitative and a Quantitative Approach to Developing a Household Food Insecurity Scale for Bangladesh. J. Nutr. 2006;136(5):1420S1430.

[12] Alvarez M, Estrada A, Montoya E, Melagr-Quininez H. Validación de Escala de la Seguridad Alimentaria Doméstica en Antioquia, Colômbia. Salud Publica de Mexico 2006;48(006):474-481.

[13] Melgar-Quinonez HR, Zubieta AC, MkNelly B, Nteziyaremye A, Gerardo MFD, Dunford C. Household Food Insecurity and Food Expenditure in Bolivia, Burkina Faso, and the Philippines. J. Nutr. 2006;136(5):1431S-1437.

[14] Pérez-Escamilla R, Randolph S, Hathie I, Gaye I. Adaptation and validation of the USDA food security scale in rural Senegal. FASEB J 2004;18(106 A abstract \# 104.1).

[15] Pérez-ESCAMILLA R, SEGALLCORREAA AM, MARANHA LK, SAMPAIO MFA, MARÍN L, PANIGASSI G. An Adapted Version of the US Departament of Agriculture Food Insecure Module Is a Valid Tool for Assessing Food Insecurity in Campinas,Brasil. Journal of Nutrition 2004;134:1923 - 1928.

[16] Segall-Corrêa AM, Perez-Escamilla R, Marin-Leon L, Yuyama L, Vianna RPT, Coitinho D, et al. Evaluation of household food insecurity in Brazil: validity assessment in diverse sociocultural settings. Concurso RedSan 2007, 2009, [cited 1; $1^{\text {a }}$ :[325]. Available http://www.rlc.fao.org/iniciativa/pdf/memre dsan.pdf

[17] Sampaio MdFA, Kepple AW, SegallCorrêa AM, Oliveira JAd, Panigassi G, Maranha LK, et al. (In)Segurança Alimentar: Experiência de grupos focais com populações rurais do Estado de São Paulo. Segurança Alimentar e Nutricional 2006;13(1):64-77.

[18] Segall-Corrêa AM, Panigassi G, Sampaio M, Marin-León L, Pérez-Escamilla R. Validación de instrumento de medida de la inseguridad alimentaria y hambre, em el contexto de las políticas brasileñas de combate el hambre. Perspectivas en Nutrición Humana 2007;2:p.89 - 102.

[19] Segall-Corrêa AM, Marin-León L, Sampaio MFA, Panigassi G, PérezEscamilla R. Insegurança Alimentar no Brasil: do Desenvolvimento dos instrumentos de medida aos primeiros resultados Nacionais. Brasília, DF: MDS; 2007.

[20] Melgar-Qinonez H, Nord M, PérezEscamilla R, Segall-Corrêa AM. Psychometric properties of a modified UShousehold food security survey module in Campinas, Brazil. European Journal of Clinical Nutrition 2007;1:245 - 257.

[21] Hackettt M, Melgar-Quinoez H, R P-E, Segall Corrêa AM. Gender of respondent does not affect the psychometric properties of the Brazilian Household Food Security Scale. Int J Epidemiology 2008;37(4):76674.

[22] Segall Corrêa AM, Perez-Escamilla R, Maranha Lk, Sampaio MdFA, Vianna RPdT, Yuyama L, et al. (IN) Segurança alimentar no Brasil: validação de metodologia para acompanhamento e avaliação. 2004 [cited Setembro de 2009]; Relatório Técnico]. Available from: http://bvsms.saude.gov.br/bvs/publicacoes/v alidacao brasill.pdf 
[23] Panigassi G, Segall-Corrêa AM, MarinLeón L, Pérez-Escamilla R, Sampaio MdFA, Maranha LK. Insegurança alimentar como indicador de iniqüidade: análise de inquérito populacional. Cadernos de Saúde Pública 2008;24:2376-2384.

[24] Leão M. Segurança Alimentar e Risco de Sobrepeso e Obesidade em famílias de crianças menores de 6 anos-. Brasília: UNB; 2005 .

[25] IBGE. Pesquisa Nacional por Amostra de Domicílios - Suplemento de Segurança Alimentar. Rio de Janeiro: IBGE; 2006.

[26] Hoffmann R. Determinantes da insegurança alimentar no Brasil: Análise dos dados da PNAD de 2004. Segurança Alimentar e Nutricional 2008;15:49-61.

[27] Segall-Corrêa AM, Marin-Leon L, Pérez-Escamilla $\mathrm{R}, \mathrm{H} \mathrm{H}$, Pacheco LMS, Souza RP. A transferência de renda no Brasil e a insegurança alimentar; analisando a PNAD 2004. Revista de Nutrição da PUCCAMP 2008;21(Supl):39 - 51.

[28] BERQUÓ E, GARCIA S, LAGO T. Dimensóes do Processo Reprodutivo e da Saúde da Criança-Pesquisa Nacional de Demografia e Saúde da Criança e da Mulher - PNDS 2006. 1a ed. Brasília: Ministério da Saúde \& CEBRAP; 2009.

[29] Segall-Corrêa AM, Leticia Leon-Marin, Giseli Panigassi, Rafael Perez-Escamilla. Segurança Alimentar em Domicílio. In: Elza Berquó, Sandra Garcia, Tânia Lago, editors. Relatório Final - Pesquisa Nacional de Demografia e Saúde da Criança e da Mulher - PNDS 2006. Brasilia: Ministério da Saúde, M S; 2008, prelo. p. 306.

[30] IPEA. PNAD 2008: Primeiras análises. Comunicado da Presidência No. 302009 [cited 2009 Setembro 2009]; Available from: http://www.ipea.gov.br/default.jsp

[31] IBGE. Síntese de indicadores sociais2007: Pesquisa Nacional Por Amostra de Domicílios. 2009[cited Setembro de 2009;
334].

Available

from: http://www.ibge.gov.br/home/estatistica/pop ulacao/trabalhoerendimento/pnad2007/sintes epnad2007.pdf

[32] Vianna RPdT, Segall-Corrêa AM. Insegurança alimentar das famílias residentes em municípios do interior do estado da Paraíba, Brasil. Revista de Nutrição 2008; 21:111s-122s.

[33] Salles-Costa R, Pereira RA, Vasconcellos MTLd, Veiga GVd, Marins VMRd, Jardim BC, et al. Associação entre fatores socioeconômicos e insegurança alimentar: estudo de base populacional na Região Metropolitana do Rio de Janeiro, Brasil. Revista de Nutrição 2008 [cited 21; 99s-109s]. Available from: http://www.scielo.br/scielo.php?script=sci_artte $\mathrm{xt} \&$ pid $=\mathrm{S} 1415-52732008000500009 \& \mathrm{nrm}=$ iso

[34] Fávaro T, Ribas D, Zorzatto J, SegallCorrêa A, Panigassi G. Segurança alimentar em famílias indígenas Teréna, Mato Grosso do Sul, Brasil. Cadernos de Saúde Pública 2007;23:785 - 793 .

[35] Yuyama LKO, Py-Daniel V, Ishikawa NK, Medeiros JF, Kepple AW, SegallCorrêa AM. Percepção e compreensão dos conceitos contidos na Escala Brasileira de Insegurança Alimentar, em comunidades indígenas no estado do Amazonas, Brasil. Revista de Nutrição 2008;21:53s-63s.

[36] Brasil. Lei Orgânica da Segurança Alimentar e Nutricional - LOSAN. In: CONSEA, editor. Brasília; 2006. 
Anexo 1

\section{SEGURANCA ALIMENTAR}

\begin{tabular}{|c|}
\hline ATENÇÃO ENTREVISTADORA (O) ESTE MÓDULO DEVE SER RESPONDIDO POR \\
PESSOA ADULTA, RESPONSÁVEL OU COM CONHECIMENTO DAS CONDIÇÕES \\
ALIMENTARES NO DOMICÍLIO \\
\hline Em todos os quesitos, você deve se referir aos ÚLTIMOS 3 MESES para orientar a \\
resposta da (o) entrevistada (o).
\end{tabular}

Agora vou ler para você algumas perguntas sobre a alimentação em sua casa. Elas podem ser parecidas umas com as outras, mas é importante que você responda a todas elas.

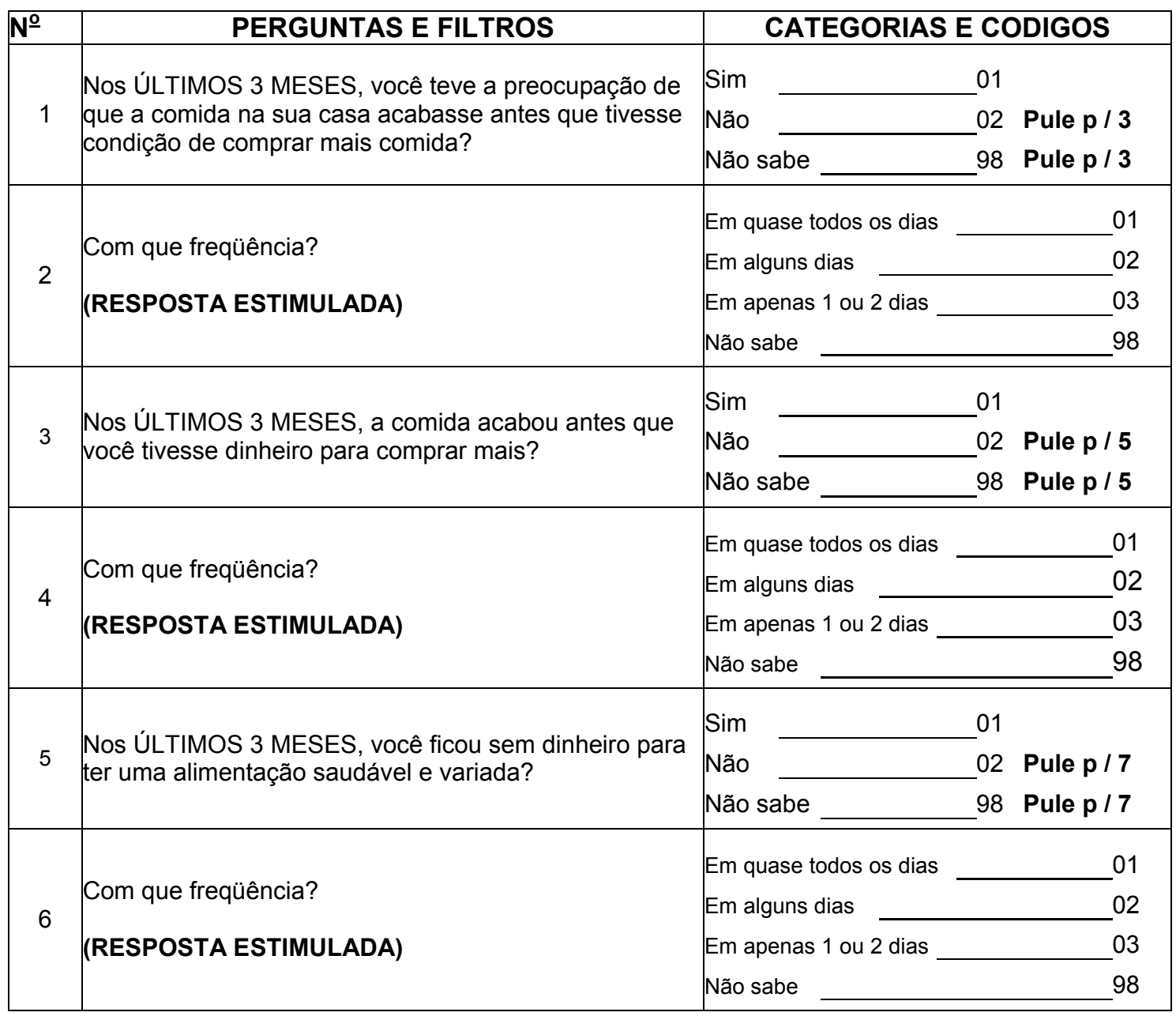




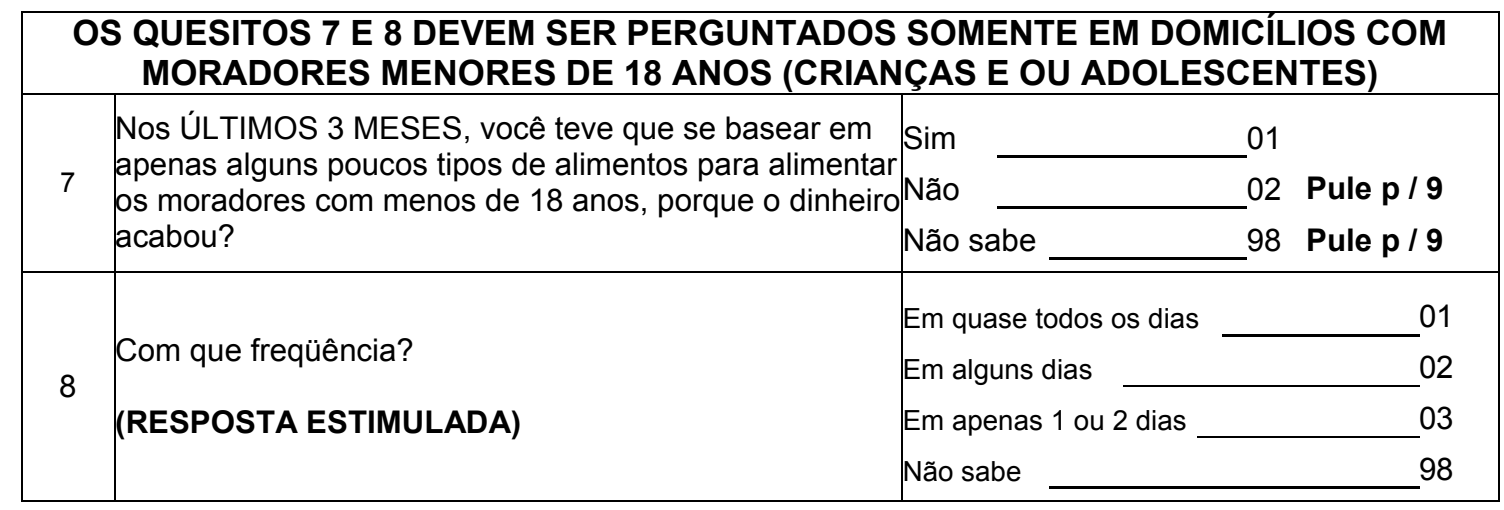

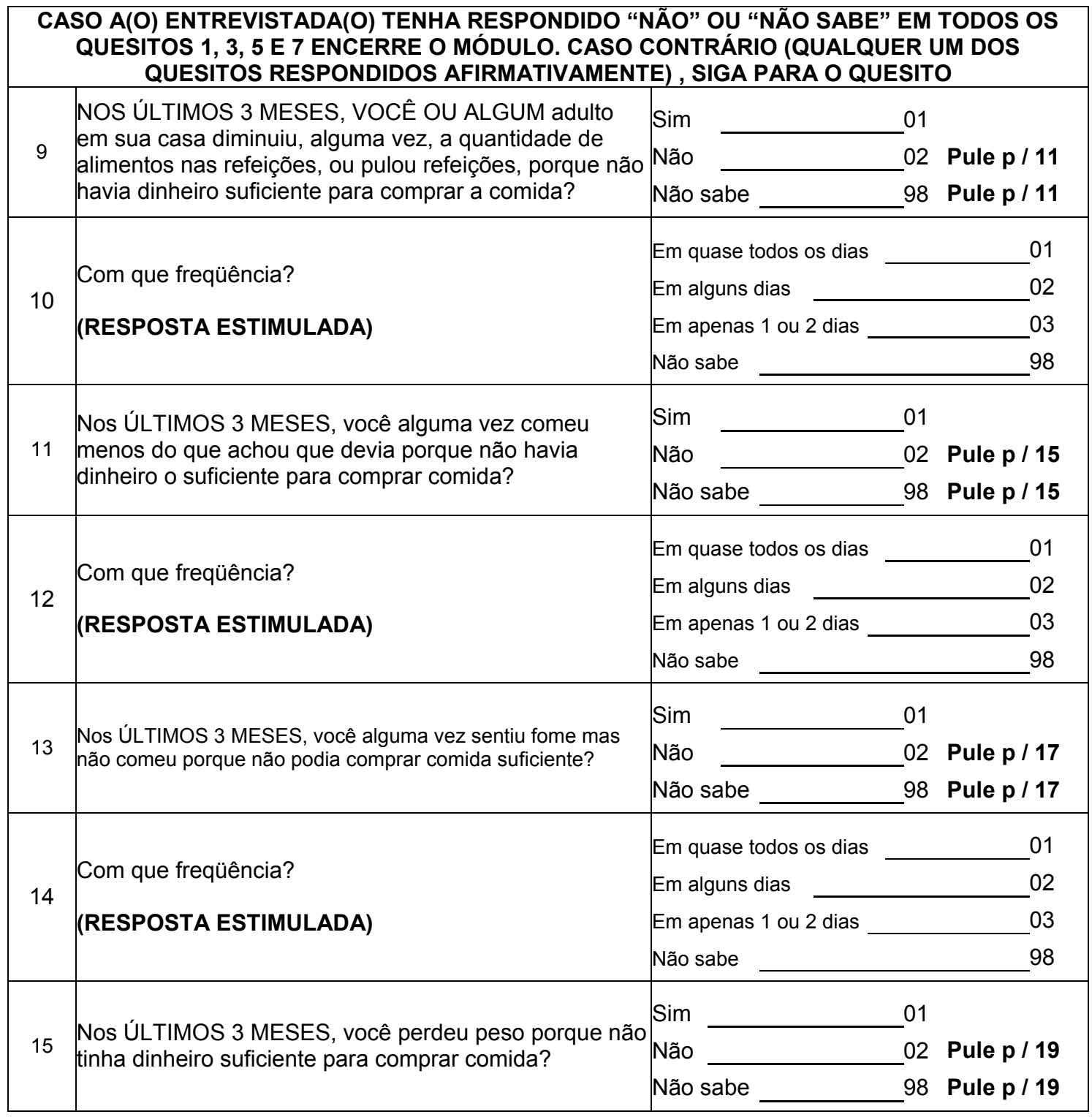




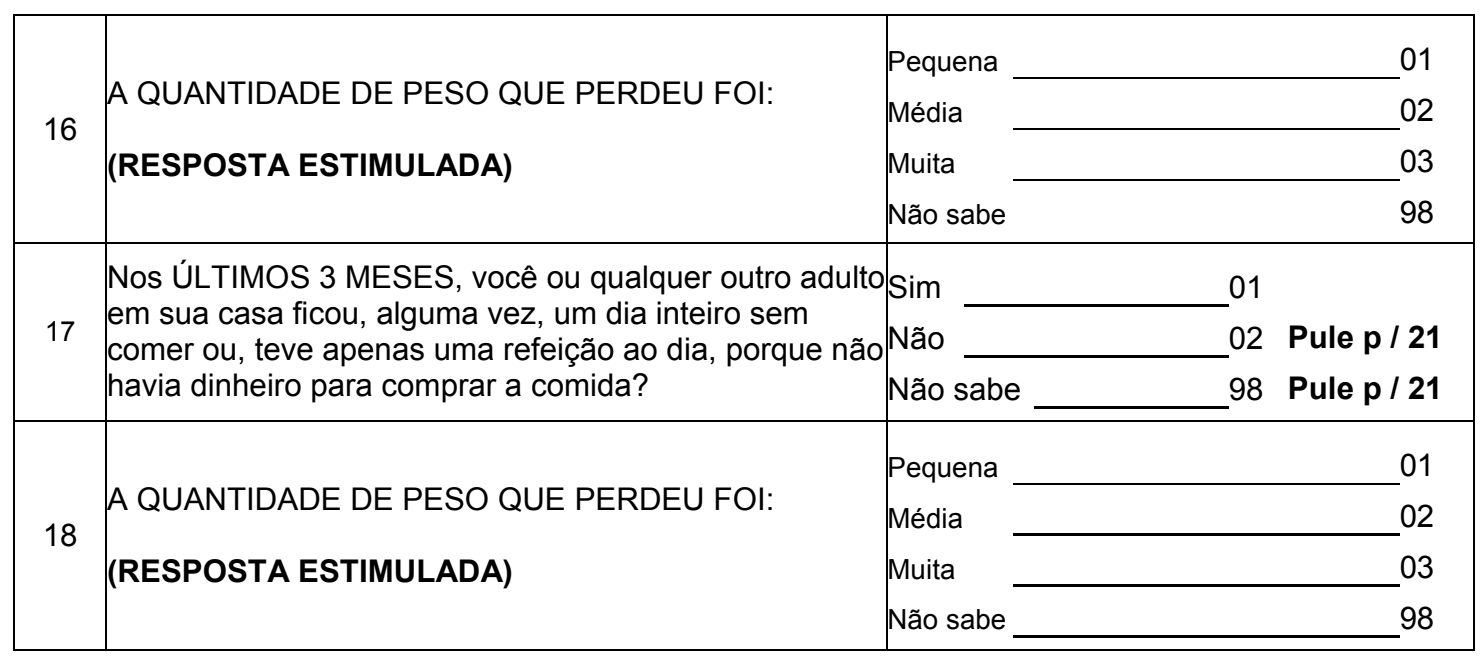

\begin{tabular}{|c|c|c|c|c|}
\hline & $\begin{array}{l}\text { OS QUESITOS ABAIXO DEVEM SER PERGUNTADOS } \\
\text { MORADORES MENORES DE } 18 \text { ANOS (CRIANC } \\
\text { SE NAO HOUVER MENORES DE } 18 \text { ANOS }\end{array}$ & $\begin{array}{l}\text { APENAS EM DOMÍC } \\
\text { CAS E/OU ADOLESC } \\
\text { ENCERRE O MÓDU }\end{array}$ & OS & ue tem \\
\hline & Nos ÚLTIMOS 3 MESES, você não pode oferecer a & Sim & 01 & \\
\hline 19 & alimentacão saudável e variada, porque não tinha & Não & 02 & Pule $\mathrm{p} / 23$ \\
\hline & dinheiro? & Não sabe & -98 & Pule p / 23 \\
\hline & & Em quase todos os dias & & 01 \\
\hline 20 & Com que frequência? & Em alguns dias & & \\
\hline & (RESPOSTA ESTIMULADA) & Em apenas 1 ou 2 dias & & _03 \\
\hline & & Não sabe & & 98 \\
\hline & Nos ÚLTIMOS 3 MESES, algum morador com menos & Sim & 01 & \\
\hline 21 & de 18 anos não comeu em quantidade suficiente, & Não & 02 & Pule $\mathrm{p} / 25$ \\
\hline & & Não sabe & -98 & Pule p / 25 \\
\hline & & Em quase todos os dias & & 01 \\
\hline 22 & fom que trequencia? & Em alguns dias & & 02 \\
\hline & (RESPOSTA ESTIMULADA) & Em apenas 1 ou 2 dias & & 03 \\
\hline & & Não sabe & & 98 \\
\hline & Nos ÚLTIMOS 3 MESES, você, alguma vez, diminuiu a & Sim & 01 & \\
\hline 23 & morador com menos de 18 anos, porque não havia & Não & 02 & Pule p / 27 \\
\hline & dinheiro suficiente para comprar a comida? & Não sabe & -98 & Pule p / 27 \\
\hline & & Em quase todos os dias & & 01 \\
\hline 24 & Com que inequencia? & Em alguns dias & & 02 \\
\hline & (RESPOSTA ESTIMULADA) & Em apenas 1 ou 2 dias & - & 03 \\
\hline & & Não sabe & & 98 \\
\hline
\end{tabular}




\begin{tabular}{|c|c|c|c|c|}
\hline \multirow{3}{*}{25} & \multirow{3}{*}{$\begin{array}{l}\text { Nos ÚLTIMOS } 3 \text { MESES, alguma vez algum morador } \\
\text { com menos de } 18 \text { anos deixou de fazer alguma } \\
\text { refeição, porque não havia dinheiro para comprar a } \\
\text { comida? }\end{array}$} & Sim & \multicolumn{2}{|l|}{01} \\
\hline & & Não & 02 & Pule p / 29 \\
\hline & & Não sabe & 98 & Pule p / 29 \\
\hline \multirow{4}{*}{26} & \multirow{4}{*}{$\begin{array}{l}\text { Com que freqüência? } \\
\text { (RESPOSTA ESTIMULADA) }\end{array}$} & \multirow{2}{*}{$\begin{array}{l}\text { Em quase todos os dias } \\
\text { Em alguns dias }\end{array}$} & & 01 \\
\hline & & & & 02 \\
\hline & & Em apenas 1 ou 2 dias & & 03 \\
\hline & & Não sabe & & -98 \\
\hline \multirow{3}{*}{27} & \multirow{3}{*}{$\begin{array}{l}\text { Nos ÚLTIMOS } 3 \text { MESES, algum morador com menos } \\
\text { de } 18 \text { anos teve fome, mas você simplesmente não } \\
\text { podia comprar mais comida? }\end{array}$} & Sim & 01 & \\
\hline & & Não & 02 & Pule $p / 31$ \\
\hline & & Não sabe & -98 & Pule $p / 31$ \\
\hline \multirow{4}{*}{28} & \multirow{4}{*}{$\begin{array}{l}\text { Com que freqüência? } \\
\text { (RESPOSTA ESTIMULADA) }\end{array}$} & \multirow{2}{*}{$\begin{array}{l}\text { Em quase todos os dias } \\
\text { Em alquns dias }\end{array}$} & & 01 \\
\hline & & & & 02 \\
\hline & & Em apenas 1 ou 2 dias & & 03 \\
\hline & & Não sabe & & -98 \\
\hline \multirow{3}{*}{29} & \multirow{3}{*}{$\begin{array}{l}\text { Nos ÚLTIMOS } 3 \text { MESES, algum morador com menos } \\
\text { de } 18 \text { anos teve apenas uma refeição ao dia, ou ficou } \\
\text { sem comer por um dia inteiro, porque não havia } \\
\text { dinheiro para comprar comida? }\end{array}$} & Sim & 01 & \\
\hline & & Não & 02 & \\
\hline & & Não sabe & 98 & \\
\hline \multirow{4}{*}{30} & \multirow{4}{*}{$\begin{array}{l}\text { Com que freqüência? } \\
\text { (RESPOSTA ESTIMULADA) }\end{array}$} & \multirow{2}{*}{$\begin{array}{l}\text { Em quase todos os dias } \\
\text { Em alguns dias }\end{array}$} & & 01 \\
\hline & & & & 02 \\
\hline & & Em apenas 1 ou 2 dias & & 03 \\
\hline & & Não sabe & & -98 \\
\hline
\end{tabular}

Escala com 15 perguntas e suas 15 respectivas freqüências quando a resposta ao item é afirmativa.

São 8 perguntas relativas, exclusivamente, aos adultos da família.

São 7 perguntas relativas, exclusivamente, aos menores de 18 anos da família.

Quando existem menores de 18 anos na família todas as 15 perguntas são dirigidas ao entrevistado.
*O questionário tem a opção de trabalhar com um esquema de filtro para interromper a entrevista quando as famílias têm pequena possibilidade de conviver com insegurança alimentar. Neste caso quando respondem negativamente a todas as perguntas $1,3,5$ e 7 (esta última elaborada para incluir no filtro famílias com menores de 18 anos), interrompe-se as entrevistas. 